\title{
Design of Classroom Intelligent Lighting System Based on SCM
}

\author{
Jiale Zheng ${ }^{1, \text { a }}$ \\ ${ }^{1}$ North China Electric Power University, Beijing, China, 102200 \\ a email
}

Keywords: Human Body Induction, Classroom Lighting, Single Chip Computer, Intelligence

\begin{abstract}
The light is the necessity of life for everybody. When the night comes, it is very inconvenient to turn on the light in the dark. And when the person leaves the house, he often forgets to turn off the lights which will cause huge waste of energy. In view of the lighting control method of classrooms, especially the current situation of the intelligent control of classroom lighting, this research analyzes the principles and realization methods of intelligent control of classroom lighting, proposes a design idea based on SCM and develops a hardware device of intelligent control system and the corresponding software. The system has the advantages of small size, convenient control, high reliability, strong specificity and reasonable price.
\end{abstract}

\section{Research Contents and Objective}

With the rapid development of intelligent technology, intelligent home appliances have become more and more popular. The classroom light control is the application of single chip computer which act as a control chip. The hardware design is divided into the sensor and the signal processing part, the central processing unit which is composed by the monolithic computer, the intelligent control classroom lamp. The paper analyzes the detailed description of the functional role of each part. The software design adopts modular structure, and the design of each module is completed. The paper for classroom lighting control, analysis of the classroom lighting intelligent control principle and realization method is proposed based on single-chip design, intelligent lighting control system of ideas, and on this basis, developed intelligent control system of hardware module and software part.

The research of classroom lighting control system can be used for the transformation of the existing classroom lighting system, which will realize the lighting system of humanized intelligent management, improve the efficiency of electricity. It also can realize the automatic and manual lighting control compatible and to reduce the cost. Therefore, the system has good reliability and practicality.

\section{Block Diagram of System}

Classroom lighting control can achieve effective classroom lighting intelligent control. The input parameters is mainly exists in the human body signal and environmental light signals and other external factors, the ambient light intensity reaches a certain value does not turn on the lights, ambient light intensity is below a certain threshold and people turn on the lights, theory and experiment prove this way to the classroom lamp intelligent control can achieve these goals. 


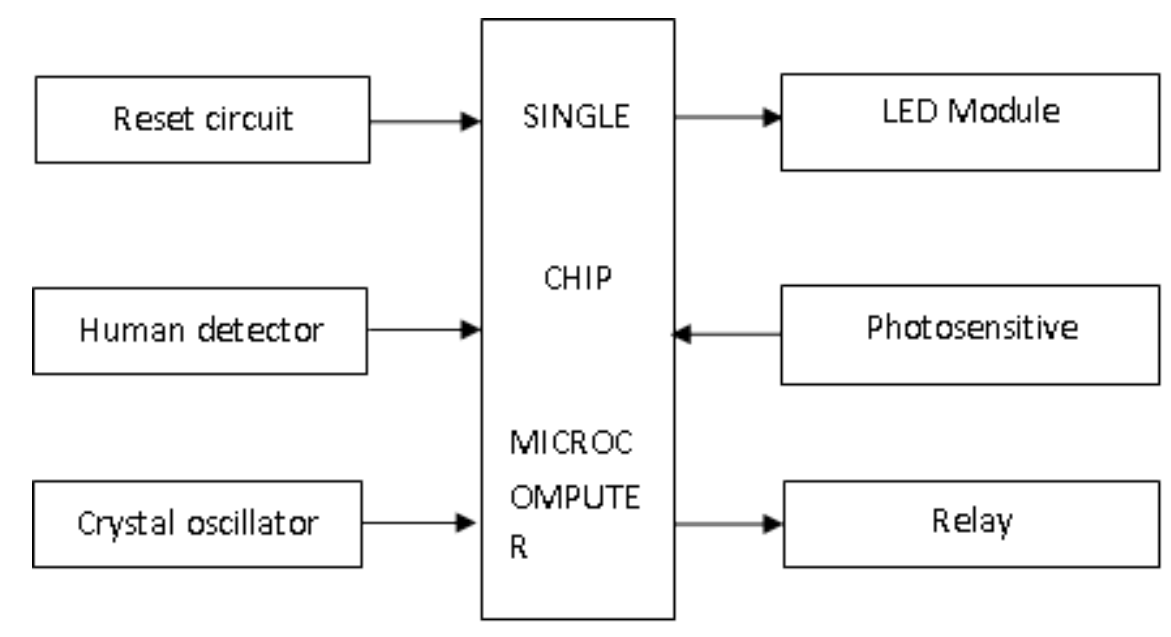

Figure 1. Block diagram of system

\section{Design of System Hardware}

Outline of System Hardware. The system taking into account the effects of environmental factors on the installed more and classroom control equipment in the human body exists sensors often park environment change and instability, so in the design process, electronic components selection, line layout and equipment placed to full consideration to the problems of anti-interference. The developed controller takes the natural light intensity and the human body as the main input parameters of the controller. Automatic and manual control can be realized. In the natural environment light strong light enough, regardless of the presence of people, did not turn on the lights; in the weak light of the natural environment, human existence and over a certain period of time controller can automatically turn on the lights. Until after leaving to delay some time off.

In this paper, the classroom lighting controller is mainly composed of two parts: hardware and software. The hardware part is the premise, which is the foundation of the whole system. It mainly provides the platform for the software to run. The software part is the signal of the hardware port, which is collected, analyzed and processed, and finally realizes the function of the controller. System control unit is to MCU control module as the core, and other peripheral circuit mainly include: a sensor module, a photosensitive module and relay driving module system power supply module, environmental light module, human exist, this design utilizes the monolithic integrated circuit, a voltage comparator, a photosensitive resistance, a human induction module, relays, etc. to constitute a light intelligent design. Design application in the classroom, library, and some other public places, when the light is dark and, the relay will pull to drive any load, intelligent delay, when no one lamp does not light, in order to achieve the effect of energy saving and intelligent.

Design of Master Module. The design and selection of STC89C5l as into simple structure of motor control chip.STC89C51 and implemented on the programmer flash electrically erasable amounted to more than tens of thousands of times. The STC89C51 is a 4K with bytes of flash programmable read only memory (FAlsh - ProgrAmmABle And ErAsABle Memory Only ReAd FPEROM) low voltage, high performance CMOS8 bit microprocessor, commonly known as single chip microcomputer. The device is fabricated by ATMEL high density nonvolatile memory manufacturing technology, which is compatible with the MCS-51 instruction set and output pin of the industrial standard.

Photosensitive resistance is also called light pipe, commonly used in the production of materials for the CDs. In addition to selenium, sulfide, lead sulfide and bismuth sulfide etc. materials. With these materials in light of specific wavelength characteristics, its resistance decreases rapidly. This 
is due to the light generated carriers are involved in conductive, in an applied electric field under the effect of the drift motion, electronic toward the power supply cathode, a hole toward the power anode, so that the resistance value of the photosensitive resistor decreased rapidly.

The tube core is a photoelectric conductor which is installed on the insulating substrate with two ohm contact electrodes. Photoconductor absorbs a photon of the photoelectric effect is limited to the illumination of the surface of thin, although carrier generation also has a few diffusion to the interior to, but the diffusion depth is limited, so the photoconductor usually made of thin layers. In order to obtain a high sensitivity, the electrode of the photosensitive resistance is generally used as a sulfur pattern,

Photosensitive resistor is made of using semiconductor photoconductive effect a resistance value with the incident light intensity and changes the resistor, also known as photoconductive detector; incident light intensity, reducing resistance, weak light incident, resistance is increased. There is another kind of incident light is weak, the resistance decreases, the incident light intensity, the resistance increases. Photo - sensitive resistors are generally used for the measurement of light, the control of light, and the conversion of light to electricity. Commonly used photosensitive resistor cadmium sulfide photosensitive resistor, it is made of semiconductor materials. Photosensitive resistor for light sensitivity (i.e. spectral characteristics) and eye of visible light (0.4 0.76) mu $\mathrm{m}$ response is very close, as long as the human eye can feel the light will cause changes in the resistance. This design using photosensitive resistance and the change of resistance of partial pressure, the voltage value to LM393 voltage comparison, through the $10 \mathrm{~K}$ can be adjustable resistor can be achieved in the purchase of light regulating the value. The inner structure of LM393 is shown as follows:

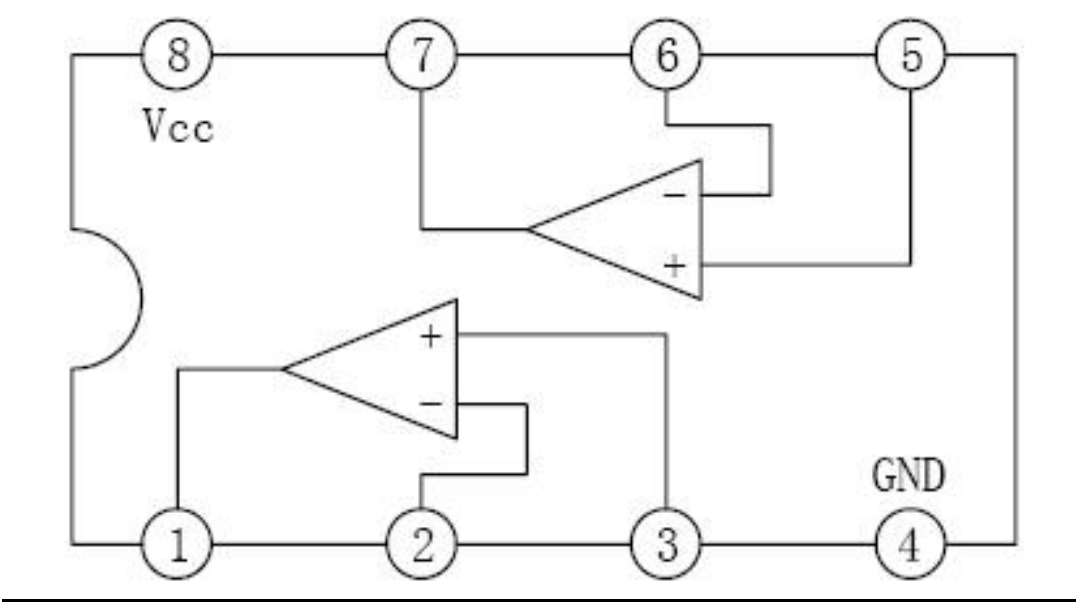

Figure 2. Inner structure of LM393

\section{Design of System Software}

When we have the command of the design of the overall understanding of the need to establish framework program flow chart, the design of module partition, module one by one, realize the function, the sub modules are reasonably connected together finally, constitutes a general program. In this chapter, the software design is based on the function of the system, which is based on the system's hardware design. The modular design idea is used to ensure the system functions. The purpose of software design is to make the MCU to load the program and to measure the distance, time and the light intensity according to the measurement results.

The flow chart of the system software is shown as follows: 


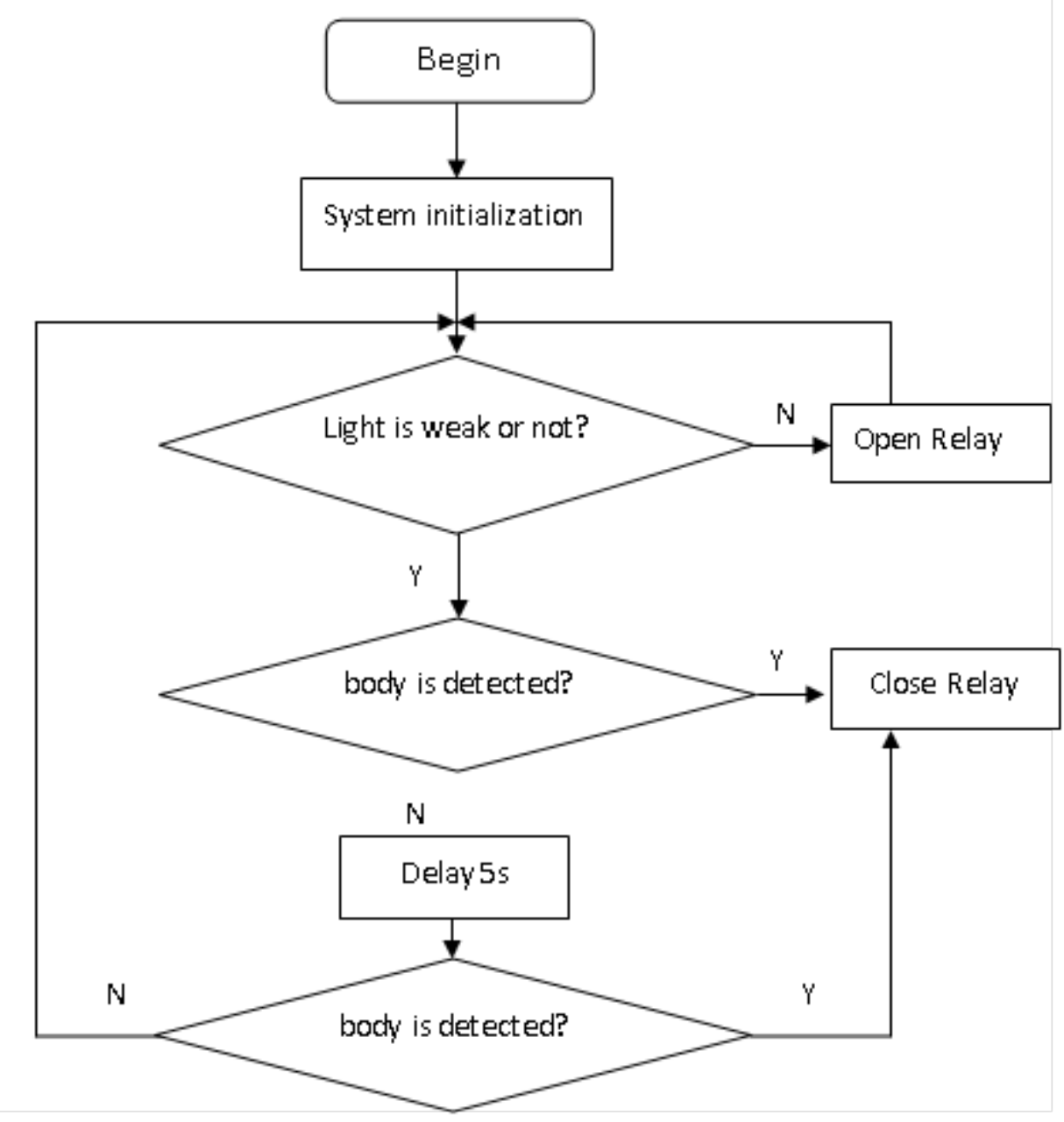

Figure 3. Flow Chart of System Software

\section{Analysis and Debugging of System}

This design is developed in Keil C environment. Keil C software support C language programming and debugging, easy to use, is the first choice for $\mathrm{C}$ language graduate. Design of the primary task is to install and learn to use this software, simple to learn and understand the Keil C, compiled through Keil C, then use STC ISP v480 software burn to the development board, to realize the connection with the kind of program. Step 1: set for stc89c52rc MCU type; the second step: open written and compiled the program file, it is with the hex file suffix; the third step: choose corresponding COM port, in my computer equipment management view the com options); the fourth step: click download and prompt please to MCU power on. Open the switch in the development board it by burning.

After the completion of program debugging and burning, the demonstration, the development board and computer connected and set the corresponding interface, power supply and download. Start after the power supply, with ten seconds of time, because the human body induction module to warm-up time. When you see the human body induction module detecting indicator is a normal light, that system can work normally, when the photosensitive detected dark environment when detected at the same time, to the human body, relay will pull, relay indicating lights. When the human body induction module cannot detect human body, the relay will be disconnected. 


\section{Conclusion and Prospect}

Taking the SCM as the core part of the control system, this paper makes use of the software programming and ultimately achieves the requirements of the classroom lighting. Preliminary test shows that the system has good applicability. It can be used in the classroom, corridors, offices, libraries and other public places.

\section{References}

[1] Cui Liangliang, Su Yanquan, Dang Xiaojing, Mechanical \& Electrical Engineering Technology, Vol. 41 (2012) No 8,p.136-138

[2] Zhang Haifeng, Li Zhenxin, Fan Hao, Zhou Yaluo, Telecom World, Vol. 13 (2016) No 3, p.273-274

[3] Zhao Liang, Lu Yun, Chen Xiaodong, Wu Li, Dou Yuan, Light \& Lighting, Vol. 36 (2012) No 2, p.36-39+55 